\title{
REALIZATIONS OF INFINITE PRODUCTS, RUELLE OPERATORS AND WAVELET FILTERS
}

\author{
DANIEL ALPAY, PALLE JORGENSEN, AND IZCHAK LEWKOWICZ
}

\section{Contents}

1. Introduction

2. Finite products

3. Infinite products

4. Markov parameters

5. Ruelle operator

6. Wavelets and rational filters

References

\begin{abstract}
Using the system theory notion of state-space realization of matrixvalued rational functions, we describe the Ruelle operator associated with wavelet filters. The resulting realization of infinite products of rational functions have the following four features: 1) It is defined in an infinite-dimensional complex domain. 2) Starting with a realization of a single rational matrix-function $M$, we show that a resulting infinite product realization obtained from $M$ takes the form of an (infinitedimensional) Toeplitz operator with the symbol that is a reflection of the initial realization for $M$. 3) Starting with a subclass of rational matrix functions, including scalar-valued ones corresponding to low-pass wavelet filters, we obtain the corresponding infinite products that realize the Fourier transforms of generators of $\mathbf{L}_{2}(\mathbb{R})$ wavelets. 4) We use both the realizations for $M$ and the corresponding infinite product to obtain a matrix representation of the Ruelle-transfer operators used in wavelet theory. By "matrix representation" we refer to the slanted (and sparse) matrix which realizes the Ruelle-transfer operator under consideration.
\end{abstract}

\section{INTRODUCTION}

Among many applications of rational matrix-valued functions are their use as filters in signal processing, and in the construction of classes of wavelets. In the latter case, the matrix function to be considered is made up of a prescribed system of scalar valued functions of a single complex variable. If $N$ is a scaling number for the wavelet under consideration, then there are associated systems of $N$ scalar-valued functions representing each of the corresponding $N$ frequency bands. Each such system produces a matrix-valued function. This particular approach to wavelet filters was considered in [9, 8, 5, 2, 11, 1]. The function corresponding to low-pass yields a father function for a wavelet when certain technical assumptions are imposed. Here we consider instead

1991 Mathematics Subject Classification. 42C40, 67T60,47A48, 40A20.

Key words and phrases. Wavelet filters, filter banks, state space realization, infinite products.

Acknowledgments: D. Alpay thanks the Earl Katz family for endowing the chair which supported his research. The authors thank the US-Israel Binational Science Foundation (BSF) Grant number 2010117. 
the matrix-valued approach: it has the advantage that it allows one to treat the combination of individual bands in a single analysis. However the issues involving infinite products in the matrix-valued case are more subtle, and we address them below. For example, to understand the infinite product formed from a rational matrix-valued function of a single complex variable, one must introduce an infinite number of complex variables. We show that, under suitable assumptions, the infinite product-function in turn then also has a realization as a function of one variable. While our motivation derived initially from the study of wavelet filters, we note that there is a host of other applications of infinite products of rational matrix functions. Indeed, the framework for our consideration of infinite products goes beyond that of wavelet filters. We shall consider these more general settings in the last section of our paper. The latter nonwavelet applications are derived from the theory of systems. Indeed the theory of realization of systems is also a key tool in our analysis of infinite products.

In [2, Section 4], we characterized wavelet filters as functions of the form

$$
M(z)=Q U\left(z^{N}\right) \Delta(z) V
$$

where

$$
V=\frac{1}{\sqrt{N}}\left(\epsilon_{N}^{-\ell j}\right)_{\ell, j=0, \ldots, N-1} \quad \epsilon_{N}:=e^{i \frac{2 \pi}{N}}
$$

is (up to scaling) the usual discrete Fourier transform matrix,

$$
\Delta(z):=\left(\begin{array}{cccc}
1 & & & \\
& z^{-1} & & \\
& & \ddots & \\
& & & z^{-(N-1)}
\end{array}\right),
$$

$U$ is a rational $(N \times N)$-valued function which takes unitary values on the unit circle, with no poles outside the closed unit disk, and and $Q$ is an arbitrary (constant) unitary matrix. One can explicitly write (1.1) as

$$
M(z)=\frac{1}{\sqrt{N}}\left(\begin{array}{cccc}
m_{0}(z) & m_{0}\left(\epsilon_{N} z\right) & \cdots & m_{0}\left(\epsilon_{N-1}^{N-1} z\right) \\
m_{1}(z) & m_{1}\left(\epsilon_{N} z\right) & \cdots & m_{1}\left(\epsilon_{N}^{N-1} z\right) \\
\vdots & & & \\
m_{N-1}(z) & m_{N-1}\left(\epsilon_{N} z\right) & \cdots & m_{N-1}\left(\epsilon_{N}^{N-1} z\right)
\end{array}\right)
$$

Note that $M(z)$ in (1.1) is unitary on $\mathbb{T}$.

An earlier relevant result on wavelet filter (1.1), (1.2) appeared in [11]. In [3] and [4], we recently further explored rectangular rational functions which are (co)isometric on the unit circle (with poles anywhere, but $\mathbb{T}$ ).

Following (1.2) one can write,

$$
\left(\begin{array}{c}
m_{0}(z) \\
m_{1}(z) \\
\vdots \\
m_{N-1}(z)
\end{array}\right)=Q U\left(z^{N}\right)\left(\begin{array}{c}
1 \\
z^{-1} \\
\vdots \\
z^{-(N-1)}
\end{array}\right)
$$

In the sequel, by choosing in (1.1)

$$
Q=(U(1) \Delta(1) V)^{*}=(U(1) V)^{*}
$$


we shall normalize the filters so that in (1.1)

$$
M(1)=I_{N} .
$$

This normalization in particular forces that the upper left entry of $M$ to satisfy

$$
m_{0}(1)=1 \text {. }
$$

This last condition is crucial to consider infinite products. For $m_{0}(z)$ in (1.2) we set:

$$
m(z):=m_{0}(z) .
$$

The wavelet father function $\varphi(w)$ is given by its Fourier transform

$$
\widehat{\varphi}(w)=\prod_{k=1}^{\infty} m\left(e^{\frac{2 \pi i w}{N^{k}}}\right) .
$$

For details, see e.g. [8] and [9].

It should be pointed out that in some engineering circles functions of the form of (1.1) are referred to as (multi-resolution) Filter Bank, whose applications transcend wavelets, see e.g. [18], [19] and even go beyond signal processing, see e.g. [16].

Here we limit our discussion to discrete time systems, where the variables, input, output, and state, are time series, that is, functions on $\mathbb{Z}$. A linear time-invariant system in this model will then be specified by a transfer matrix $M(z)$, also called a transfer function. It is a rational matrix-valued function of a single complex variable. Moreover the complex variable $z$ is dual to time, and so it represents frequency. If $M(z)$ has no pole at infinity (following engineering literature), a corresponding state space realization is any quadruple of matrices $A, B, C$, and $D$ of appropriate size, such that

$$
M(z)=D+C(z I-A)^{-1} B
$$

holds.

We assume that $M$ in (1.1), (1.2) is a matrix-valued rational function analytic at infinity, while for $m(z)$, its upper left entry, we introduce a state space realization

$$
m(z)=D+C(z I-A)^{-1} B
$$

where we can assume that the realization is minimal (that is, the size of $A$ is the smallest possible one), and that in particular $A$ has no spectrum on the unit circle since $M$ is analytic on the unit circle. Our use of the term realization conforms to its common use in the theory of systems from the study of dynamical systems and filters in engineering, and pioneered by Kalman and others; see [14, 15, 17]. Aspects of realization of filter bank as in (1.1), (1.2) were already addressed in [11], [18] and [19].

The paper consists of five sections besides the introduction, and its outline is as follows. In Section 2 we introduce state-space realization formulas of finite products of rational functions, each of a different variable. Infinite products are considered in Section 3 , As we will see in that section an important role is played by the Toeplitz operator with the related symbol equal to

$$
A+z B(I-z D)^{-1} C
$$


In Section 4, we compute the Markov parameters associated with $|m(z)|^{2}$ in terms of the given realization of $m$. In Section 5, we study the Ruelle operator and connections with rational wavelet filters are studied in Section 6.

\section{Finite PROduCtS}

As is well known, see e.g [6], [14, Section 6.4], [15], [17, Section 6.5], every $(p \times q)$-valued rational function $R(z)$ analytic at infinity can be written as

$$
R(z)=D+C(z I-A)^{-1} B,
$$

for matrices $A, B, C$ and $D$ of appropriate sizes. Equation (2.1) is called a realization of $R$ and we shall sometimes use the abbreviated form

$$
\left(\begin{array}{l|l}
A & B \\
\hline C & D
\end{array}\right)
$$

In general, the realization is highly non unique. When the dimension of the state space, (i.e. the upper left block $A$ is say $d \times d$ ), is minimal, the realization is unique up to a similarity matrix, meaning that the only freedom in the choice of the realization is

$$
\left(\begin{array}{cc}
A & B \\
C & D
\end{array}\right) \mapsto\left(\begin{array}{cc}
T & 0 \\
0 & I_{p}
\end{array}\right)\left(\begin{array}{cc}
A & B \\
C & D
\end{array}\right)\left(\begin{array}{cc}
T^{-1} & 0 \\
0 & I_{q}
\end{array}\right),
$$

where $T \in \mathbb{C}^{d \times d}$ is an arbitrary invertible matrix and where $D$ is assumed to belong to $\mathbb{C}^{p \times q}$. An important formula for the realization of the product is given in the next lemma:

Lemma 2.1. Let $R_{1}$ and $R_{2}$ be two matrix-valued rational functions analytic at infinity, and with realizations

$$
R_{1}(z)=D_{1}+C_{1}\left(z I_{n_{1}}-A_{1}\right)^{-1} B_{1} \quad \text { and } \quad R_{2}(z)=D_{2}+C_{2}\left(z I_{n_{2}}-A_{2}\right)^{-1} B_{2} .
$$

Assume that the product $R_{1} R_{2}$ makes sense. Then a realization of $R_{1}(z) R_{2}(z)$ is compactly given by:

$$
\left(\begin{array}{cc|c}
A_{1} & B_{1} C_{2} & B_{1} D_{2} \\
& A_{2} & B_{2} \\
\hline C_{1} & D_{1} C_{2} & D_{1} D_{2}
\end{array}\right)
$$

An important tool in our argument is the counterpart of (2.4) when each function depends on a different variable. See Lemma 2.3.

Factorization of rational matrix-valued functions of one variable is classical. In contrast, factorization theory of rational functions of several complex variables $z_{1}, \ldots, z_{u}$ is not well developed. However, here we consider matrix-valued rational functions of the form

$$
M(z)=M_{1}\left(z_{1}\right) M_{2}\left(z_{2}\right) \cdots M_{u}\left(z_{u}\right)
$$

where $M_{1}, \ldots, M_{u}$ are matrix-valued rational functions of appropriate sizes and analytic at infinity.

For future reference we mention the following result, whose proof is a direct verification, and will be omitted. 
Lemma 2.2. Let $\mathcal{H}_{1}$ and $\mathcal{H}_{2}$ be two Hilbert spaces and let $a: \mathcal{H}_{1} \rightarrow \mathcal{H}_{1}, b: \mathcal{H}_{2} \rightarrow$ $\mathcal{H}_{2}$, and $c: \mathcal{H}_{2} \rightarrow \mathcal{H}_{1}$ be bounded linear operators, with a and $b$ invertible. Then

$$
\left(\begin{array}{cc}
a & -c \\
0 & b
\end{array}\right)^{-1}=\left(\begin{array}{cc}
a^{-1} & a^{-1} c b^{-1} \\
0 & b^{-1}
\end{array}\right)
$$

The following very simple lemma is the key to the formulas we develop:

\section{Lemma 2.3.}

$$
\begin{array}{r}
\left(D_{1}+C_{1}\left(z_{1} I_{n_{1}}-A_{1}\right)^{-1} B_{1}\right)\left(D_{2}+C_{2}\left(z_{2} I_{n_{2}}-A_{2}\right)^{-1} B_{2}\right)= \\
=D+C(\Lambda(z)-A)^{-1} B
\end{array}
$$

where,

$$
\Lambda(z):=\left(\begin{array}{ll}
z_{1} I_{n_{1}} & \\
& z_{2} I_{n_{2}}
\end{array}\right)
$$

and the realization array (2.2) takes the form,

$$
\left(\begin{array}{cc|c}
A_{1} & B_{1} C_{2} & B_{1} D_{2} \\
& A_{2} & B_{2} \\
\hline C_{1} & D_{1} C_{2} & D_{1} D_{2}
\end{array}\right)
$$

Proof. We first note that by Lemma 2.2 we have:

$$
\begin{aligned}
(\Lambda(z)-A)^{-1} & =\left(\begin{array}{cc}
z_{1} I_{n_{1}}-A_{1} & -B_{1} C_{2} \\
0 & z_{2} I_{n_{2}}-A_{2}
\end{array}\right)^{-1} \\
& =\left(\begin{array}{cc}
\left(z_{1} I_{n_{1}}-A_{1}\right)^{-1} & \left(z_{1} I_{n_{1}}-A_{1}\right)^{-1} B_{1} C_{2}\left(z_{2} I_{n_{2}}-A_{2}\right)^{-1} \\
0 & \left(z_{2} I_{n_{2}}-z_{2} A_{2}\right)^{-1}
\end{array}\right) .
\end{aligned}
$$

Therefore we have

$$
\begin{aligned}
& D+C(\Lambda(z)-A)^{-1} B=D_{1} D_{2}+ \\
& +\left(\begin{array}{ll}
C_{1} & D_{1} C_{2}
\end{array}\right)\left(\begin{array}{cc}
\left(z_{1} I_{n_{1}}-z_{1} A_{1}\right)^{-1} & \left(z_{1} I_{n_{1}}-A_{1}\right)^{-1} B_{1} C_{2}\left(z_{2} I_{n_{2}}-A_{2}\right)^{-1} \\
0 & \left(z_{2} I_{n_{2}}-A_{2}\right)^{-1}
\end{array}\right)\left(\begin{array}{c}
B_{1} D_{2} \\
B_{2}
\end{array}\right)
\end{aligned}
$$

which is exactly the left side of (2.6).

This formula can now be iterated to obtain a realization for a product (2.5). With

$$
M_{j}\left(z_{j}\right)=D_{j}+C_{j}\left(z_{j} I_{n_{j}}-A_{j}\right)^{-1} B_{j}, \quad j=1, \ldots u,
$$

and $M(z)=M_{1}\left(z_{1}\right) M_{2}\left(z_{2}\right) \cdots M_{u}\left(z_{u}\right)$ we have

$$
M(z)=D+C(\Lambda(z)-A)^{-1} B
$$

where

$$
\Lambda(z):=\left(\begin{array}{cccc}
z_{1} I_{n_{1}} & & & \\
& z_{2} I_{n_{2}} & & \\
& & \ddots & \\
& & & z_{u} I_{n_{u}}
\end{array}\right)
$$


where $M(\infty)=\lim _{z \rightarrow \infty} M(z)$ and the realization array (2.2) takes the form,

$$
\left(\begin{array}{cccccc|c}
A_{1} & B_{1} C_{2} & B_{1} D_{2} C_{3} & \cdots & B_{1} D_{2} \cdots D_{u-2} C_{u-1} & B_{1} D_{2} \cdots D_{u-1} C_{u} & B_{1} D_{2} \cdots D_{u} \\
& A_{2} & B_{2} C_{3} & \cdots & B_{2} D_{3} \cdots D_{u-2} C_{u-1} & B_{2} D_{3} \cdots D_{u-1} C_{u} & B_{2} D_{3} \cdots D_{u} \\
& & & \ddots & & & \\
& & & & A_{u-1} & B_{u-1} C_{u} & B_{u-1} D_{u} \\
& & & & & A_{u} & B_{u} \\
\hline C_{1} & D_{1} C_{2} & D_{1} D_{2} C_{3} & \cdots & D_{1} \cdots D_{u-2} C_{u-1} & \left.D_{1} \cdots D_{u-1} C_{u}\right) & D_{1} \cdots D_{u}
\end{array}\right)
$$

We note that the case where all the functions vanish at infinity, i.e. where $\lim _{z \rightarrow \infty} M(z)=0$, leads to very simple formulas, which we gather in the following lemma.

Lemma 2.4. It holds that

$$
C_{1}\left(z_{1} I_{n_{1}}-A_{1}\right)^{-1} B_{1} C_{2}\left(z_{2} I_{n_{2}}-A_{2}\right)^{-1} B_{2} \cdots C_{u}\left(z_{u} I_{n_{u}}-A_{u}\right)^{-1} B_{u}=C(\Lambda(z)-A)^{-1} B,
$$

where $\Lambda(z)$ is as in (2.7) and the realization array (2.2) takes the form,

$$
\left(\begin{array}{cccccc|c}
A_{1} & B_{1} C_{2} & 0 & \cdots & & 0 & 0 \\
& A_{2} & B_{2} C_{3} & 0 & \ldots & 0 & 0 \\
& & & \ddots & & & \vdots \\
& & & & A_{u-1} & B_{u-1} C_{u} & 0 \\
& & & & & A_{u} & B_{u} \\
\hline C_{1} & 0 & & \cdots & & 0 & 0
\end{array}\right) .
$$

The significance of the realizations in (2.9) and in Lemma 2.4, goes beyond the scope of this work. In the sequel, we actually exploit a special case of it. See also Remark 6.4

\section{INFINITE PRODUCTS}

While the framework of realizations is typically formulated for finite matrices, (as we point out below) a number of the results make sense for infinite matrices, hence for linear operators in Hilbert space. A case in point is the realizations we obtain now for our infinite products. We now wish to let $u \rightarrow \infty$ in (2.9) when all the functions $R_{j}$ coincide:

$$
R_{1}(z)=R_{2}(z)=\cdots=M(z),
$$

where $M(z)$ is a matrix-valued rational function, analytic at infinity, with realization

$$
M(z)=D+C(z I-A)^{-1} B .
$$

We assume that $1 \geq\|M(z)\|$ for all $z \in \mathbb{T}$.

Theorem 3.1. Given a square $M(z)$ in (3.1).

(i) Assume that

$$
\lim _{k \rightarrow \infty}\left\|D^{k}\right\|^{1 / k}<1 .
$$


Then, the operators

$$
\begin{aligned}
\mathscr{A} & =\left(\begin{array}{ccccc}
A & B C & B D C & B D^{2} C & \cdots \\
0 & A & B C & B D C & \cdots \\
0 & 0 & A & B C & \cdots \\
&
\end{array}\right), \quad \ell_{2}(\mathbb{N}) \otimes \mathbb{C}^{m} \Longrightarrow \ell_{2}(\mathbb{N}) \otimes \mathbb{C}^{m} \\
\mathscr{B} & =\left(\begin{array}{c}
\vdots \\
B D^{2} \\
B D \\
B
\end{array}\right), \\
\mathscr{C} & =\left(\begin{array}{ccc}
C & D C & D^{2} C
\end{array}\right), \quad \ell_{2}(\mathbb{N}) \otimes \mathbb{C}^{m} \longrightarrow \mathbb{C}_{2}(\mathbb{N}) \otimes \mathbb{C}^{m}
\end{aligned}
$$

are bounded.

(ii) $\mathscr{A}$ in (3.3) is the block-Toeplitz operator with symbol

$$
A+z B(I-z D)^{-1} C \text {. }
$$

Proof. We have

$$
A+z B(I-z D)^{-1} C=A+z B C+z^{2} B D C+z^{3} B D^{2} C+\cdots
$$

and hence the function $\phi(z)=A+z B(I-z D)^{-1} C$ is the symbol of the block Toeplitz operator (3.3). We note that, in view of (3.2)

$$
\|\mathscr{A}\|=\left\|A+z B(I-z D)^{-1} C\right\|_{\infty}<\infty,
$$

and so the block Toepliz operator $\mathscr{A}$ is bounded. We use the fact that a block Toeplitz operator with symbol $\phi(z)$ has norm $\|\phi\|_{\infty}$; see [12].

Remark 3.2. The same result holds mutatis mutandis when instead of (3.1), the alternative realization $M(z)=D+z C(I-z A)^{-1} B$ is chosen.

Remark 3.3. Recall that

$$
\mathscr{B}=\mathcal{O}(D, B) \quad \text { and } \quad \mathscr{C}=\mathcal{C}(D, C)
$$

in (3.4) and (3.5) are the observability and controllability operators (see e.g. [14, Section 6.2], [17])

Theorem 3.4. Assume that $M(z)$ in (3.1) has no singularity at the point $z=1$ and that $M(1)=I$, and let $\left(z_{k}\right)_{k \in \mathbb{N}_{0}}$ be a sequence of complex numbers which are not poles of $M$ and such that

Then it holds that

$$
\sum_{k=0}^{\infty}\left|1-z_{k}\right|<\infty
$$

$$
\prod_{k=1}^{\infty} M\left(z_{k}\right)=\mathscr{C}(\Lambda(z)-\mathscr{A})^{-1} \mathscr{B}
$$

where $\mathscr{A}, \mathscr{B}$ and $\mathscr{C}$ are defined by $(3.3)$ - 3.5 , and where

$$
\Lambda(z)=\left(\begin{array}{ccc}
z_{1} I_{n} & & \\
& z_{2} I_{n} & \\
& & \ddots
\end{array}\right) .
$$


Proof. Since the realization of $M$ is assumed minimal, 1 is not in the spectrum of $A$ and we have $M(1)=D+C\left(I_{n}-A\right)^{-1} B$. Thus

$$
\begin{aligned}
M(z)-M(1) & =D+C\left(z I_{n}-A\right)^{-1} B-D-C\left(I_{n}-A\right)^{-1} B \\
& =C\left(z I_{n}-A\right)^{-1} B-C\left(I_{n}-A\right)^{-1} B \\
& =(1-z) C(z I-A)^{-1}(I-A)^{-1} B .
\end{aligned}
$$

Furthermore, (3.7) implies in particular that $\lim _{k \rightarrow \infty} z_{k}=1$. Let

$$
K_{0}=\max _{z \in V}\left\{\left\|C\left(z I_{n}-A\right)^{-1}\left(I_{n}-A\right)^{-1} B\right\|\right\},
$$

where $V$ is a closed neighborhood of 1 in which $m$ has no pole, and let

$$
K=\max _{\text {where the } z_{u} \notin V}\left\{K_{0},\left\|C\left(z_{u} I_{n}-A\right)^{-1}\left(I_{n}-A\right)^{-1} B\right\|\right\}
$$

Then we have

$$
\left\|M\left(z_{k}\right)-I\right\| \leq K \cdot\left|1-z_{k}\right| .
$$

Therefore the series $\sum_{k=1}^{\infty}\left\|I-M\left(z_{k}\right)\right\|$ and hence the product $\prod_{k=1}^{\infty} M\left(z_{k}\right)$ are convergent. The equality (3.8) is now easy to verify.

Corollary 3.5. Assume that $M(1)=I$, and let $\left(\theta_{k}\right)$ be a sequence of numbers on the real line such that

$$
\sum_{k=0}^{\infty}\left|\theta_{k}\right|<\infty .
$$

Then the infinite product

$$
\prod_{k=0}^{\infty} M\left(e^{i t \theta_{k}}\right), \quad t \in \mathbb{R}
$$

converges for all real $t$.

Proof. Since $\left|e^{i \theta}-1\right| \leq|\theta|$ for $\theta$ real, we have

$$
\left\|M\left(e^{i t \theta_{k}}\right)-I\right\| \leq K_{1} \cdot\left|\theta_{k}\right|
$$

where now we can take

$$
K_{1}=\max _{\theta \in[0,2 \pi]}\left\|C\left(e^{i \theta} I_{n}-A\right)^{-1}\left(I_{n}-A\right)^{-1} B\right\|,
$$

and hence the result.

Corollary 3.6. In the notation and hypothesis of Corollary 3.5, the product

$$
\prod_{k=0}^{\infty} M\left(e^{i \frac{2 \pi w}{N^{k}}}\right)
$$

converges for every $w \in \mathbb{R}$. 


\section{Markov parameters}

Let $M$ be analytic in the exterior and on the boundary of the unit disk, with minimal realization $M(z)=D+C(z I-A)^{-1} B$. In particular $\sigma(A) \subset \mathbb{D}$. Let $h_{0}+\sum_{k=1}^{\infty} \frac{h_{k}}{z^{k}}$ be the Laurent expansion at infinity of $M$. The coefficients $h_{0}, h_{1}, \ldots$ are called the Markov parameters of $M$, see e.g. [14, Subsections 5.1.2, 6.2.1], [17, Section 6.5]. They are given by $h_{0}=D$ and

$$
h_{k}=C A^{k-1} B, \quad k=1,2, \ldots
$$

We extend the sequence $h_{k}$ by

$$
h_{u}=0, \quad u<0 .
$$

Since the spectral radius of $A$ is strictly less than 1 , we can set

$$
\Gamma=\sum_{u=0}^{\infty} A^{* u} C^{*} C A^{u} .
$$

Note that $\Gamma$ is called the observability Gramian, and is the unique solution of the Stein equation

$$
\Gamma-A^{*} \Gamma A=C^{*} C
$$

We set

$$
Y=D^{*} C+B^{*} \Gamma A .
$$

In view of the next result we recall that a rational function $r$ with no poles on the unit circle belongs to the Wiener algebra of the disk, that is, can be written as

$$
r(z)=\sum_{n \in \mathbb{Z}} z^{n} r_{n}
$$

where $\sum_{n \in \mathbb{Z}}\left|r_{n}\right|<\infty$. See for instance [13, Corollary 3.2].

Theorem 4.1. Let $\left(c_{n}\right)_{n \in \mathbb{Z}}$ be defined by

$$
\|M(z)\|^{2}=\sum_{n \in \mathbb{Z}} c_{n} z^{n}, \quad z \in \mathbb{T} .
$$

Then

$$
c_{n}=\sum_{j \in \mathbb{Z}} h_{j}^{*} h_{j+n}, \quad n \in \mathbb{Z} .
$$

Therefore,

$$
c_{n}=\left\{\begin{array}{lc}
B^{*} A^{*(-n-1)} Y^{*}, & n<0 \\
D^{*} D+B^{*} \Gamma B, & n=0 \\
Y A^{n-1} B, & n>0
\end{array}\right.
$$

Proof. For $n=0$ we have

$$
\begin{aligned}
c_{0} & =D^{*} D+\sum_{k=1}^{\infty} B^{*} A^{*(k-1)} C^{*} C A^{k-1} B \\
& =D^{*} D+B^{*}\left(\sum_{k=1}^{\infty} A^{*(k-1)} C^{*} C A^{k-1}\right) B \\
& =D^{*} D+B^{*} \Gamma B,
\end{aligned}
$$


where $\Gamma$ is the Gramian matrix from (4.2).

We now assume $n>0$. Then,

$$
\begin{aligned}
c_{n} & =h_{0}^{*} h_{n}+\sum_{k=1}^{\infty} h_{k}^{*} h_{k+n} \\
& =D^{*} C A^{n-1} B+\sum_{k=1}^{\infty} B^{*} A^{k-1} C^{*} C A^{k+n-1} B \\
& =D^{*} C A^{n-1} B+B^{*} \Gamma A^{n} B .
\end{aligned}
$$

Finally, for $n<0$, we have:

$$
\begin{aligned}
c_{n} & =\sum_{k=-n}^{\infty} h_{k}^{*} h_{k+n} \\
& =\sum_{u=0}^{\infty} h_{u-n}^{*} h_{u} \\
& =B^{*} A^{*(-n-1)} C^{*} D+\sum_{u=1}^{\infty} B^{*} A^{*(u-n-1)} C^{*} C A^{u-1} B \\
& =B^{*} A^{*(-n-1)} C^{*} D+B^{*} A^{*(-n)} \Gamma B .
\end{aligned}
$$

Corollary 4.2. Let $d$ be the size of $A$ (that is, $A \in \mathbb{C}^{d \times d}$ ). Let $Y$ be defined by (4.3) and

$$
\mathcal{C}(A, B)=\left(\begin{array}{lllll}
B & A B & A^{2} B & \cdots & A^{d-1} B
\end{array}\right)
$$

Then

$$
c_{n}=Y A^{n-1} B, \quad n=1, \ldots
$$

and

$$
\left(\begin{array}{llll}
c_{1} & c_{2} & \cdots & c_{d}
\end{array}\right)=Y \cdot \mathcal{C}(A, B) .
$$

Theorem 4.3. There exists complex numbers $a_{0}, \ldots, a_{d-1}$ such that

$$
a_{0} c_{1}+a_{1} c_{2}+\cdots+a_{d-1} c_{d}+c_{d+1}=0,
$$

and more generally, for any $p \geq 1$,

$$
a_{0} c_{p}+a_{1} c_{p+1}+\cdots+a_{d-1} c_{d+p-1}+c_{d+p}=0 .
$$

Proof. By the Cayley-Hamilton theorem there exists numbers $a_{0}, a_{1}, \ldots, a_{d-1}$ such that

$$
a_{0}+a_{1} A+a_{2} A^{2}+\cdots+a_{d-1} A^{d-1}+A^{d}=0 .
$$

It then follows from (4.6) that we have (4.8), and more generally (4.9).

Formulas (4.5) take a simpler form in a number of cases, which we mention as remarks:

Remark 4.4. If $A$ is nilpotent (and then $m$ is a polynomial in $1 / z$ ) we have

$$
c_{d+1}=c_{d+2}=\cdots=0 .
$$


Remark 4.5. Assuming that $D=0$ implies that in (4.3) $Y=D^{*} C$ and (4.5) becomes,

$$
c_{n}= \begin{cases}B^{*} A^{*(-n)} \Gamma B, & n \leq 0, \\ B^{*} \Gamma A^{n} B, & n>0 .\end{cases}
$$

Remark 4.6. We recall that the observability Gramian $\Gamma$, see (4.2), is invertible if and only if the pair $(C, A)$ is observable, meaning that

$$
\cap_{u=1}^{d-1} \operatorname{ker} A^{u}=\{0\}
$$

Then one assume $\Gamma=I_{d}$ by taking $T=\Gamma^{1 / 2}$ as similarity matrix in (2.3). When furthermore $D=0$ we then have

$$
c_{n}= \begin{cases}B^{*} A^{*(-n)} B, & n \leq 0 \\ B^{*} A^{n} B, & n>0 .\end{cases}
$$

Proposition 4.7. Assume $M$ is rational. Then the coefficients $c_{n}$ satisfy the estimates of the form

$$
\left|c_{k}\right| \leq C e^{-\alpha|k|}, \quad k \in \mathbb{Z}
$$

for every $\alpha>\rho(A)$, where $\rho(A)$ is the spectral radius of $A$.

\section{Ruelle operator}

Using $m(z)$ from (1.5), (1.7), the associated Ruelle operator (or transfer operator) is defined by (see [8, §3.2])

$$
(R f)(z)=\frac{1}{N} \sum_{\substack{w \in \mathbb{T} \\ w^{N}=z}}|m(w)|^{2} f(w) .
$$

See [8, p. 156]. In terms of the coefficients (4.4) in Theorem 4.1 it is the operator between appropriate subspaces of $\ell_{2}(\mathbb{Z})$ and with matrix representation

$$
r_{\ell, j}=\frac{1}{N} c_{N \ell-j},
$$

that is

$$
(R f)_{\ell}=\frac{1}{N} \sum_{k \in \mathbb{Z}} c_{N \ell-k} f_{k}
$$

Example 5.1. When $N=2, D=0$ and $\Gamma=I_{d}$ the matrix representation of the Ruelle operator is

$$
\frac{1}{2}\left(\begin{array}{lccccccc}
\cdots & & & & & & \ldots \\
\cdots & B^{*} B & B^{*} A B & B^{*} A^{* 2} B & B^{*} A^{* 3} B & B^{*} A^{* 4} B & & \ldots \\
\cdots & B^{*} A^{2} B & B^{*} A B & B^{*} B & B^{*} A^{*} B & B^{*} A^{* 2} B & B^{*} A^{* 3} B & \ldots \\
\cdots & B^{*} A^{4} B & B^{*} A^{3} B & B^{*} A^{2} B & B^{*} A B & B^{*} B & B^{*} A^{*} B & \ldots \\
\cdots & & & & & & & \ldots
\end{array}\right)
$$

where the box denotes the $(0,0)$ element (see (4.11)).

Let

$$
\mathscr{E}_{r}=\left\{\left(f_{n}\right)_{n \in \mathbb{Z}} ;\|f\|_{r, 1} \stackrel{\text { def. }}{=} \sum_{n \in \mathbb{Z}} e^{r|n|}\left|f_{n}\right|<\infty\right\}
$$


and

$$
\mathscr{E}_{r}^{(2)}=\left\{\left(f_{n}\right)_{n \in \mathbb{Z}} ;\|f\|_{r, 2}^{2} \stackrel{\text { def. }}{=} \sum_{n \in \mathbb{Z}} e^{r|n|}\left|f_{n}\right|^{2}<\infty\right\}
$$

See [20], 8, p. 158] for these last spaces. We note that an element of $\mathscr{E}_{r}$ satisfies an estimate of the form

$$
\left|f_{n}\right| \leq K e^{-r|n|}, \quad n \in \mathbb{Z}
$$

for some $K>0$.

Theorem 5.2. Assume that the coefficients $c_{n}$ satisfy the estimate (4.12). Then for every choice of $\beta$ and $\beta^{\prime}$ such that

$$
\alpha<\beta \quad \text { and } \quad \beta^{\prime}<N \alpha
$$

the Ruelle operator is continuous from $\mathscr{E}_{\beta}$ into $\mathscr{E}_{\beta^{\prime}}$ and from $\mathscr{E}_{\beta}^{(2)}$ into $\mathscr{E}_{\beta^{\prime}}^{(2)}$.

Proof. Let $f=\left(f_{n}\right)_{n \in \mathbb{Z}}$ be an element of $\mathscr{E}_{\beta}$, with $\beta$ as in (5.4). The $\ell$ component of the vector $R f$ is given by (5.2),

$$
(R f)_{\ell}=\frac{1}{N} \sum_{k \in \mathbb{Z}} c_{N \ell-k} f_{k}
$$

and, using (4.12) and (5.3) can be estimated as:

$$
\begin{aligned}
N\left|(R f)_{\ell}\right| & \leq \sum_{k \in \mathbb{Z}}\left|c_{N \ell-k}\right| \cdot\left|f_{k}\right| \\
& \leq \sum_{k \in \mathbb{Z}} C e^{-\alpha|N \ell-k|} \cdot K e^{-\beta|k|} \\
& =\sum_{k \in \mathbb{Z}} C K e^{-\alpha(|N \ell-k|+|k|)} \cdot e^{(\alpha-\beta)|k|} \\
& \leq C K e^{-\alpha|N \ell|} \sum_{k \in \mathbb{Z}} e^{(\alpha-\beta)|k|} .
\end{aligned}
$$

Hence for any $\beta^{\prime}<N \alpha$,

$$
\left.\sum_{\ell \in \mathbb{Z}} \mid(R f)_{\ell}\right) \mid \cdot e^{\beta^{\prime}|\ell|}<\infty
$$

and so $R f \in \mathscr{E}_{\beta^{\prime}}$. 
Let now $\epsilon>0$ and $f, g \in \mathscr{E}_{\beta}$ and such that $\|f-g\|_{\beta, 1}<\epsilon$. Then in a way similar to the above argument we write

$$
\begin{aligned}
N\|f-g\|_{\beta^{\prime}, 1} & =\sum_{\ell \in \mathbb{Z}}\left(\sum_{k \in \mathbb{Z}}\left|c_{N \ell-k}\right| \cdot\left|f_{k}-g_{k}\right|\right) \\
& \leq \sum_{\ell \in \mathbb{Z}}(\sum_{k \in \mathbb{Z}} C e^{-\alpha|N \ell-k|} \cdot e^{-\alpha|k|} \underbrace{e^{\left(\alpha-\beta^{\prime}\right)|k|}}_{\text {is }<1} e^{\beta^{\prime}|k|}\left|f_{k}-g_{k}\right|) \\
& \leq \sum_{\ell \in \mathbb{Z}}\left(\sum_{k \in \mathbb{Z}} C e^{-\alpha|N \ell|} \cdot e^{\beta^{\prime}|k|}\left|f_{k}-g_{k}\right|\right) \\
& =C\left(\sum_{\ell \in \mathbb{Z}} e^{-\alpha|N \ell|}\right) \cdot\|f-g\|_{\beta^{\prime}, 1} .
\end{aligned}
$$

and continuity of $R$ follows.

The case of the spaces $\mathscr{E}_{\beta}^{(2)}$ and $\mathscr{E}_{\beta^{\prime}}^{(2)}$ is proved in the same way.

For a result related to the following theorem in the non rational case, see [8, p. 158].

The first item in the next theorem is taken from [8, p.156-159], [10].

Theorem 5.3. Let $m(z)$ be as in (1.5), (1.7).

(1) The Ruelle operator has finite trace, and its trace is given by the formula

$$
\operatorname{Tr} R=\frac{1}{N} \sum_{k \in \mathbb{Z}} c_{(N-1) k}=\frac{1}{N} \sum_{\substack{w \in \mathbb{T} \\ w^{N}=1}}|m(w)|^{2} .
$$

(2) In the rational case, we have

$$
\operatorname{Tr} R=\frac{1}{N}\left(D D^{*}+B^{*} \Gamma B+Y(I-A)^{-1} B^{*}+B\left(I-A^{*}\right)^{-1} Y^{*}\right) .
$$

Proof. The second item is a direct consequence of formulas (4.5) .

\section{WAVELETS AND RATIONAL FILTERS}

In this section, we show that starting from a rational wavelet filter, the infinite product (1.6) is indeed in $\mathbf{L}_{2}(\mathbb{R}, d x)$. To that purpose it is enough to prove that

$$
R 1 \leq 1
$$

for the corresponding Ruelle operator, where, by definition of $R$,

$$
(R 1)(z)=\frac{1}{N} \sum_{\substack{w \in \mathbb{T} \\ w^{N}=z}}|m(w)|^{2},
$$

with $m$ from (1.5), (1.7).

Let $M$ be a rational wavelet filter, that is a function of the form (1.1). Recall that its first column is given by (1.3).

Proposition 6.1. It holds that

$$
R 1=1
$$


Proof. From (1.3) and (1.4) we have that

$$
m(z)=\sum_{j=1}^{N} z^{1-j}\left[W\left(z^{N}\right)\right]_{1 j}
$$

where $[W]_{j k}$ denotes the $j k$ entry in a matrix $W$, with

$$
W\left(z^{N}\right):=(U(1) V)^{*} U\left(z^{N}\right) .
$$

Thus, for $z \in \mathbb{T}$,

$$
\begin{aligned}
\frac{1}{N} \sum_{\substack{\omega \in \mathbb{T} \\
\omega^{N}=z}}|m(\omega)|^{2} & =\frac{1}{N} \sum_{\substack{\omega \in \mathbb{T} \\
\omega^{N}=z}}\left|\sum_{j=1}^{N} \omega^{1-j}\left[W\left(\omega^{N}\right)\right]_{1 j}\right|^{2} \\
& =\frac{1}{N} \sum_{\substack{\omega \in \mathbb{T} \\
\omega^{N}=z}} \sum_{j, k=1}^{N} \omega^{-j+k}[W(z)]_{1 j} \overline{[W(z)]_{1 k}} \quad\left(\text { note that } \omega^{N}=z\right) \\
& =\sum_{j, k=1}^{N} \delta_{j k}[W(z)]_{1 j} \overline{[W(z)]_{1 k}} \quad\left(\text { since } \frac{1}{N} \underset{\substack{\omega \in \mathbb{T} \\
\omega^{N}=z}}{\left.\sum^{-j+k}=\delta_{j k}\right)}\right. \\
& \left.=\sum_{j=1}^{N}[W(z)]_{1 j} \overline{[W(z)]_{1 j}}=1 \quad \text { (since } W \text { takes unitary values on } \mathbb{T}\right) .
\end{aligned}
$$

Theorem 6.2. Let $m$ be the upper left entry of a wavelet filter, see (1.5), (1.7). Then the infinite product (1.6) converges to an $\mathbf{L}_{2}(\mathbb{R})$ function.

Proof. We proceed in a number of steps.

STEP 1: The infinite product (1.6) converges pointwise.

This follows from Corollary 3.5 with $\theta_{k}=\frac{2 \pi w}{N^{k}}$ since $M(1)=I_{N}$ and hence $m(1)=1$.

We now set

$$
f_{k}(w)=1_{\left[-\frac{N^{k}}{2}, \frac{N^{k}}{2}\right]}(w) \prod_{\ell=0}^{k} m\left(e^{i \frac{2 \pi w}{N^{\ell}}}\right), \quad k=0,1, \ldots
$$

The key to the proof is to establish the identity

$$
\int_{-\frac{N^{k}}{2}}^{\frac{N^{k}}{2}}\left|f_{k}(w)\right|^{2} d w=1
$$

STEP 2: (6.3) holds: 
Indeed,

$$
\begin{aligned}
\int_{-\frac{N^{k}}{2}}^{\frac{N^{k}}{2}}\left|f_{k}(w)\right|^{2} d w & =\int_{-\frac{N^{k}}{2}}^{\frac{N^{k}}{2}}\left(\prod_{\ell=0}^{k}\left|m\left(e^{i \frac{2 \pi w}{N^{\ell}}}\right)\right|^{2}\right) d w \\
& =\int_{-\frac{N^{k-1}}{2}}^{\frac{N^{k-1}}{2}}\left(\prod_{\ell=0}^{k-1}\left|m\left(e^{i \frac{2 \pi w}{N^{\ell}}}\right)\right|^{2}\right) \underbrace{\left(\sum_{p=0}^{N-1}\left|m\left(e^{2 \pi i \frac{w+p N^{k-1}}{N^{k}}}\right)\right|^{2}\right)}_{\text {This is } R 1} d w \\
& =\int_{-\frac{N^{k-1}}{2}}^{\frac{N^{k-1}}{2}}\left(\prod_{\ell=0}^{k-1}\left|m\left(e^{i \frac{2 \pi w}{N^{\ell}}}\right)\right|^{2}\right) d w \quad(\text { since } R 1=1) \\
& =\int_{-\frac{N^{k-1}}{2}}^{\frac{N^{k-1}}{2}}\left|f_{k-1}(w)\right|^{2} d w \quad \text { (by definition of } f_{k-1} \text {; see (6.2) ). }
\end{aligned}
$$

So we have

$$
\int_{-\frac{N^{k}}{2}}^{\frac{N^{k}}{2}}\left|f_{k}(w)\right|^{2} d w=\int_{-\frac{N^{k-1}}{2}}^{\frac{N^{k-1}}{2}}\left|f_{k-1}(w)\right|^{2} d w .
$$

Iterating this equality we get to

$$
\begin{gathered}
\int_{-\frac{N^{k}}{2}}^{\frac{N^{k}}{2}}\left|f_{k}(w)\right|^{2} d w=\int_{-\frac{N^{k-1}}{2}}^{\frac{N^{k-1}}{2}}\left|f_{k-1}(w)\right|^{2} d w=\cdots \\
\cdots=\int_{-\frac{1}{2}}^{\frac{1}{2}}\left|f_{0}(w)\right|^{2} d w=\int_{-\frac{1}{2}}^{\frac{1}{2}}\left|m\left(e^{2 \pi i w}\right)\right|^{2} d w<\infty
\end{gathered}
$$

STEP 3: The pointwise limit function $f(w)=\prod_{\ell=1}^{\infty} m\left(e^{2 \pi i \frac{w}{N^{\ell}}}\right)$ belongs to $\mathbf{L}_{2}(\mathbb{R})$. Indeed, by Fatou's lemma,

$$
\begin{aligned}
\int_{\mathbb{R}}|f(w)|^{2} d w & =\int_{\mathbb{R}} \lim _{k \rightarrow \infty}\left|f_{k}(w)\right|^{2} d w \\
& =\int_{\mathbb{R}} \liminf _{k \rightarrow \infty}\left|f_{k}(w)\right|^{2} d w \\
& \leq \liminf _{k \rightarrow \infty} \int_{\mathbb{R}}\left|f_{k}(w)\right|^{2} d w<\infty
\end{aligned}
$$

in view of the previous step.

Remark 6.3. The preceding arguments hold still in the case $R 1 \leq 1$. This covers important cases of rational filters for which the function $U$ in (1.1) is only contractive as opposed to unitary.

Remark 6.4. In future work we shall use the multivariable state space techniques introduced in Section 2 to extend our results to the multivariable case, including the case of an infinite number of variables, and to study connections with infinite dimensional analysis (see [7] for the latter).

Acknowledgments: It is a pleasure to thank the referees for a very careful reading of the manuscript. 


\section{REFERENCES}

[1] D. Alpay, P. Jorgensen, and I. Lewkowicz. "Extending wavelet filters: infinite dimensions, the non-rational case, and indefinite inner product spaces". In Excursions in harmonic analysis. Volume 2, Appl. Numer. Harmon. Anal., pages 69-111. Birkhäuser/Springer, New York, 2013.

[2] D. Alpay, P. Jorgensen, and I. Lewkowicz. "Parametrizations of all wavelet filters: input-output and state-space". Sampling Theory in Signal and Image Processing (STSIP), 12:159-188, 2013.

[3] D. Alpay, P.E.T Jorgensen, and I. Lewkowicz "Characterizations of Families of Rectangular, Finite Impulse Response, Para-Unitary Systems", a preprint. Available at http://arxiv.org/abs/1410.0280

[4] D. Alpay, P.E.T Jorgensen, and I. Lewkowicz "Characterizations of Rectangular, (Para)Unitary Rational Functions", a manuscript. Available at http://arxiv.org/abs/1410.0283

[5] D. Alpay, P. Jorgensen, I. Lewkowicz, and I. Marziano. "Representation formulas for Hardy space functions through the Cuntz relations and new interpolation problems". In Xiaoping Shen and Ahmed Zayed, editors, Multiscale signal analysis and modeling, pages 161-182. Springer, 2013.

[6] H. Bart, I. Gohberg, and M.A. Kaashoek. Minimal factorization of matrix and operator functions, volume 1 of Operator Theory: Advances and Applications. Birkhäuser Verlag, Basel, 1979.

[7] F. Biagini, Y. Hu, B. Øksendal, and T. Zhang. Stochastic calculus for fractional Brownian motion and applications. Probability and its Applications (New York). Springer-Verlag London Ltd., London, 2008.

[8] O. Bratteli and P. Jorgensen. Wavelets through a looking glass. Applied and Numerical Harmonic Analysis. Birkhäuser Boston Inc., Boston, MA, 2002.

[9] O. Bratteli and P. Jorgensen. Wavelet filters and infinite-dimensional unitary groups. In Wavelet analysis and applications (Guangzhou, 1999), volume 25 of AMS/IP Stud. Adv. Math., pages 35-65. Amer. Math. Soc., Providence, RI, 2002.

[10] I. Daubechies. "Using Fredholm determinants to estimate the smoothness of refinable functions". In Approximation theory VIII, Vol. 2 (College Station, TX, 1995), volume 6 of Ser. Approx. Decompos., pages 89-112. World Sci. Publ., River Edge, NJ, 1995.

[11] X. Gao, T.Q. Nguyen and G. Strang, "On Factorization of $M$-Channel Paraunitary Filterbanks", IEEE Trans. Signal Proc., Vol. 49, pp. 1433-1446, 2001.

[12] I. Gohberg, S. Goldberg, and M. Kaashoek. Classes of linear operators. Vol. I, volume 49 of Operator Theory: Advances and Applications. Birkhäuser Verlag, Basel, 1990.

[13] I. Gohberg and M.A. Kaashoek. Block Toeplitz operators with rational symbols. In I. Gohberg, J.W. Helton, and L. Rodman, editors, Contributions to operator theory and its applications (Mesa, AZ, 1987), volume 35 of Oper. Theory Adv. Appl., pages 385-440. Birkhäuser, Basel, 1988.

[14] T. Kailath. Linear systems. Prentice-Hall Inc., Englewood Cliffs, N.J., 1980.

[15] R. E. Kalman, P. L. Falb, and M. A. Arbib. Topics in mathematical system theory. McGraw-Hill Book Co., New York, 1969.

[16] Y-P. Lin, S-M. Phoong and P.P. Vaidyanathan, Filter Bank Transceivers for OFDM and DMT Systems, Cambridge University Press, 2011.

[17] E. Sontag. Mathematical control theory, volume 6 of Texts in Applied Mathematics. SpringerVerlag, New York, second edition, 1998. Deterministic finite-dimensional systems.

[18] J. Tuqan and P.P. Vaidyanathan, "A State Space Approach to the Design of Globally Optimal FIR Energy Compaction Filters", IEEE Trans. Sig. Proc., Vol. 48, pp. 2822-2838, 2000.

[19] P.P. Vaidyanathan, Multirate Systems and Filter Banks, Prentice-Hall, Signal Processing Series, 1993.

[20] S. J. L. van Eijndhoven and J. L. H. Meyers. "New orthogonality relations for the Hermite polynomials and related Hilbert spaces". J. Math. Anal. Appl., 146(1):89-98, 1990. 
(DA) Department of MATHEMATiCS

BenGurion University OF THE NEGEV

BEER-SHEVA 84105 ISRAEL

E-mail address: dany@math.bgu.ac.il

(PJ) Department of Mathematics

$14 \mathrm{MLH}$

The UNIVERSITY OF IOWA

IowA City, IA 52242-1419 USA

E-mail address: palle-jorgensen@uiowa.edu

(IL) Department of Electrical Engineering

Ben Gurion University of the Negev

P.O.B. 653,

BE'ER SHEva 84105,

ISRAEL

E-mail address: izchak@ee.bgu.ac.il 